\title{
Bloch's law and a temporal integration model for simple reaction time to light*
}

\author{
JAMES D. HILDRETH \\ 1877 Grand Avenue, St. Paul, Minnesota 55105
}

\begin{abstract}
In a series of experiments designed to determine whether Bloch's law holds for simple RT to low-energy visual stimuli, mean RTs were found to agree with Bloch's law to a close approximation only when a narrow range of stimulus intensities is used. However, they could be accounted for more generally by (1) assuming that detection depends on a "visual response function" (VRF) initiated and maintained by the light stimulus (when the time integral of the VRF reaches a criterion, $S$ detects the light and initiates a response); and (2) the fact that VRF generated by a square-wave flash rises quickly to its maximum, remains at this value for the duration of the flash. and then decays exponentially to zero after flash offset. S continues to integrate the VRF throughout its lifetime, and consequently for a brief stimulus, detection will occur during the exponentially decaying portion of the response-the portion corresponding to "visual persistence." Finally. when luminances used vary by more than a factor of four, Bloch's law fails to hold, while the model succeeds, implying that the temporal integration model more generally accounts for RTs.
\end{abstract}

Although luminance-duration reciprocity (constant behavioral effect to stimuli with the same luminance $x$ duration product) has been observed in many threshold experiments, e.g., Sperling \& Jolliffe (1965), since Bloch's classical work (Bloch, 1885), the use of RT as the dependent variable in studies of both luminance and duration dates from an experiment by Raab and Fehrer (1962) in which intensities well above threshold were used. While some less extensive reports exist, e.g., Lewis (1964) and Sticht (1969), only two other major investigations of this general type have appeared: a set of experiments at threshold intensities by Grossberg (1968) and a set by Kietzman and Gillam (1972). In the Raab and Fehrer and the Grossberg studies, luminance was fixed within any single block of trials and stimulus duration randomly varied over trials within a block. And in both studies, the conclusion was essentially the same: Bloch's law (that luminance-duration reciprocity exists for durations less than a critical time period) does not hold in general for RTs. Grossberg reported, however, that Bloch's law (BL) held approximately if RT was measured from the end (offset) of the flash. Grossberg's discussion of a limited test by Lewis (1964) in an unpublished $\mathrm{PhD}$ dissertation suggested the hypothesis that the asymmetrical treatment of the two independent variables (luminance and duration) was responsible for the negative Raab and Fehrer result. Indeed, this was Lewis's conclusion from his own experiment, in which two flashes of equal energy, interspersed randomly among trials of the experiment, led to similar RT distributions. When only duration is randomized, adaptation level, temporal expectancy, and response set

*Work on this project was supported by the National Institute of Mental Health (Predoctoral Fellowships 1-FO1-MH45396-01 and 4-FO1-MH45396-02). I thank John Yellott, Jr. for suggesting the terminology "visual response function" (VRF) and "temporal integration model," for his editorial advice in the writing of this paper, and for his encouragement and assistance throughout this research. Thanks are also due David LaBerge for the use of his laboratory at the University of Minnesota. where this research was conducted. may vary systematically between groups of trials of fixed luminance, confounding any attempt to demonstrate reciprocity. This suggests that Grossberg's failure to obtain reciprocity over the range of stimulus energy used may also have been due to the same asymmetry in experimental design.

This interpretation is supported both by our results (reported beiow) and a recent report by Kietzman and Gillam (1972). The latter authors designed their first experiment to counter exactly this difficulty of asymmetry. They compared effects on RT of equivalent stimulus intensity and duration combinations, avoiding manipulation of only stimulus duration. Their results supported the conclusion that the BL holds for foveal light flashes of durations up to $11 \mathrm{msec}$. This rather low limiting duration may have been a result of the short ( 5 min) dark adaptation period used. Our results (below) indicate (1) that BL holds at least up to $32 \mathrm{msec}$, when the set of luminances used spans a factor of 4 , (2) that BL fails when the set of luminances used spans a factor of 10. The failure of BL in the latter case might be due to loss of the state of dark adaptation caused by the highest intensities, as the experiment progresses from trial to trial. Certainly, one would expect trial-to-trial effects if intensity varied over a very wide range.

\section{RT, Brightness, and VER Latency}

Credit for discovering the relationship between a component of response latency and brightness is apparently due to $\mathrm{H}$. Pieron, who, from his experiments on perceptual latency (Liang \& Pieron, 1954) and RT and perceived brightness (Pieron, 1920, 1952), suggested that response speed, defined as the reciprocal of the reducible segment of response latency $\left(T-T_{0}\right)$, should be directly proportional to brightness. (By $T_{o}$ is meant a positive constant time with the restriction $T_{0}<T$. By $T$. we mean the total response latency.) Brightness (S) has been shown in many magnitude estimation experiments, 
e.g., Hanes (1949), Hopkinson (1956), Onley (1961), Raab (1962), and Stevens and Galanter (1957), to be closely fit by a power function of physical intensity ( $\mathrm{S}=$ $\left({ }^{\beta}\right)$. The exponent $(\beta)$ of the power function in the case of a visual stimulus ranges from about .3 to .5 , depending on angular size (large to small, respectively) and retinal position (peripheral to foveal, respectively), and has been recognized to be about one-third, as a general rule of thumb, cf. Stevens and Galanter (1957). Pieron concluded from his results that speed of visual response also varies in approximate proportion to the cube root of stimulus intensity.

Vaughn (1964) provided the first explicit neurophysiological demonstration of this relationship by measuring the latency of the visual evoked response (VER) as a function of stimulus intensity. Vaughn observed that the logarithm of the reduced VER latency, $T-T_{0}$, as a function of the logarithm of stimulus energy was a linear function with a slope of about one-third. Taken together with the results of magnitude estimation experiments, this result supports Pieron's contention that "response speed" (the reciprocal of the reduced latency) is directly proportional to brightness; both are described by power functions of physical intensity with exponents of about one-third.

The power law approach was used by Vaughn et al (1966) in a later experiment, in which the latencies of the VER and RT were compared concurrently as a function of stimulus intensity ( $\ell$ ). Both were well fit by power functions of intensity with exponents varying from -.29 to -.44 . It was concluded that the source of this variation was mainly retinal on the basis of studies of human ERG amplitude (Armington et al, 1960), of the latency of discharge of the Limulus ommatidium (Hartline, 1934), and optic nerve of the conger eel (Adrian \& Matthews, 1927), data of the kind typically fitted by others with the function $[a+b \log (\ell)]-1$. These data are well fit by power functions with exponents near one-third; cf. Vaughn et al (1966). It therefore appears that power functions with exponents of about one-third are capable of fitting the same kind of data well fit by reciprocal linear functions of $\log (\ell)$. Further, if brightness is proportional to $\left(T-T_{0}\right)^{-1}$ (reciprocal reduced latency), these two apparently conflicting representations parallel the historical psychophysical functions for scaling sensation: Fechner's logarithmic scale implies that visual brightness is a linear function of $\log (\ell)$, while Stevens's power law states that brightness is proportional to $\ell^{\beta}$, where $\beta=$ 0.33 . Evidently these functions are similar over narrow ranges of $\ell$ when the exponent $\beta$ is near one-third.

Although the exact mathematical relation of reciprocal reduced latency $\left(T-T_{0}\right)^{-1}$ and brightness to physical stimulus intensity is not specified in the temporal integration model to be described below, we will show below that this model is compatible with Bloch's law for RTs and that the power law relation between $r_{\ell}$ and $\ell$ must be approximately proportional both to $\log (\ell)$ and to $\ell^{\beta}-1$, where the exponent $\beta$ must be near one-third, as was found in our experiments.

In summary, there is considerable evidence to support Pieron's hypothesis that the reciprocal reduced latency of visual detection as measured by RT and VER latency is inversely proportional to brightness as measured by the method of magnitude estimation (see also Teichner \& Krebs, 1972). It is tempting to infer from this that temporal summation of transduced luminance (i.e., "brightness") up to a threshold value determines the (reduced) latency of detection. The discussion below of a class of temporal integration models is a formal development of this idea. These models assume that the light stimulus gives rise to a "visual response function" (VRF), the amplitude of which is an increasing function of stimulus luminance (e.g., a power function). The exponential decay model, a member of this class, makes the additional assumption that the VRF decays exponentially following stimulus offset, a sort of visual persistence. It is assumed that a detector mechanism integrates the VRF, including the decay trace, until, with (reduced) latency $\mathrm{T}-\mathrm{T}_{\mathbf{o}}$, a threshold value is reached.

Of course, if this threshold is never reached, detection cannot occur. Specification of a VRF and a randomly varying threshold therefore allows theoretical predictions about threshold data, although such are beyond the scope of this paper.

\section{METHOD \\ Procedure}

The purpose of the series of three experiments reported here was to investigate simple RT as a function of stimulus luminance and duration. A question of particular interest was whether BL holds for simple RT when complete symmetry is maintained between the independent variables of luminance and duration, i.e., when both are presented randomly. Essentially, each experimental session consisted of a sequence of simple RT trials, $25 \%$ of which were catch (no stimulus) trials. Over the remaining $75 \%$ of the trials, stimulus duration and luminance varied randomly, as described below. Altogether, there were three experiments, corresponding to three different ranges of luminance. Except for differences in luminance values, these experiments were identical in procedure, and the same two $S s$ participated in all three.

The order in which the experiments were run was: for $S 1-2$, 3,1 ; for $S 2-1,2,3$. Ss were extensively practiced before each experiment with new luminance parameters. About six 1-h sessions were typically required to reach asymptotic performance for a single experiment, after which six 1 -h data collection sessions were conducted for each.

\section{Stimuli}

Stimuli were produced with two fluorescent lamps (GE F 8T5 Daylight) in each of three fields of a Scientific Prototype tachistoscope. Each field provided one of the luminance levels. The circuits produced nearly rectangular stimuli, which was confirmed by photographs of the electrical output of a photocell. Stimulus duration was controlled by a small computer (CDC 160) and was tested to an accuracy of within. $1 \mathrm{msec}$ at the electronic gate of the tachistoscope. Durations used in all 
experiments were $1,2,4,8,16,32,64$, and $128 \mathrm{msec}$. Stimulus luminance was controlled by neutral density filters calibrated with a Photovolt photometer (520ME). The stimulus fields were congruent, circular, subtended $1.6 \mathrm{deg}$. and appeared white when transilluminated by the lamp. A dim red dot, 1 deg below the stimulus, served as a fixation point. The S. dark-adapted for $1 / 2 \mathrm{~h}$, sat in a dark room and viewed binocularly. Head position was controlled by a rubber viewport. The response button was a sensitive microswitch mounted vertically below a handrest and and closed by pulling action.

\section{Instructions}

Instructions to the $S$ were to respond as quickly as possible to the light flashes while maintaining a false alarm (FA) rate of less than $4 \%$.

\section{Event Sequence}

The content and duration of fixed foreperiods and stimulus periods, stimulus randomization and selection, latency measurement and recording, and conditional feedback were controlled by the computer. The trial event sequence, contingent feedback, and sequencing control are shown in Fig. 1. This sequence applied on every trial. Because of the critical importance of maintaining equal stimulus characteristics among fields, and because onset reliability for short-duration stimuli made it necessary, each trial contained a special bulb warm-up period of $300 \mathrm{msec}$ in all three fields. The resulting flash was masked from S's view by a shutter. The warm-up had the effect of minimizing sequential dependencies in latencies due to lamp temperature, and of increasing onset reliability at $1-\mathrm{msec}$ durations to $100 \%$.

\section{Subjects}

The author served as $S 1$. A paid male college senior served as $\$ 2$. Both $\mathrm{S} s$ served in all three experiments.

Trlal lreat sequence

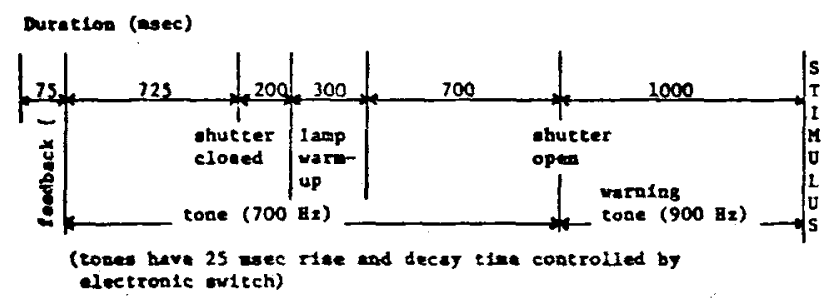

Concingene Peedbeck and Sequencios Cootrol:

\begin{tabular}{|c|c|c|c|}
\hline & & $\begin{array}{l}\text { response } \\
\text { vithin } \\
1 \text { eecond }\end{array}$ & $\begin{array}{l}\text { no response } \\
\text { uthin } \\
1 \text { second }\end{array}$ \\
\hline$(758)$ & $\begin{array}{l}\text { light } \\
\text { eximuluo }\end{array}$ & $\begin{array}{l}\text { cootinue } \\
\text { to next } \\
\text { trial }\end{array}$ & $\begin{array}{l}\text { Miss } \\
\text { (white nolae } \\
\text { feedbeck) } \\
\text { cont. to bext trial }\end{array}$ \\
\hline (258) & $\begin{array}{l}\text { cotech } \\
\text { eriel }\end{array}$ & $\begin{array}{l}\text { FALSE ALAPM } \\
\text { (apeech noise } \\
\text { feadbeck) } 6 \\
\text { coat. to bext } \\
\text { trial }\end{array}$ & $\begin{array}{l}\text { continue } \\
\text { to next } \\
\text { crial (after } \\
1 \text { econd) }\end{array}$ \\
\hline
\end{tabular}

Fig. 1. The trial event sequence included fixed foreperiods with tones on each trial. Each stimulus or catch trial was followed by feedback contingent on the S's response.
Table 1

Luminances by Experiment Number

\begin{tabular}{cccc}
$\begin{array}{c}\text { Experi- } \\
\text { ment }\end{array}$ & \multicolumn{3}{c}{ Luminances Used (fL) } \\
\hline 1 & .600 & .300 & .150 \\
2 & .150 & .075 & .038 \\
3 & .038 & .019 & .009 \\
& \multicolumn{2}{c}{ Luminances in Terms of $\mathrm{L}=.6 \mathrm{fL}$} \\
1 & $\mathrm{~L}$ & $\mathrm{~L} / 2$ & $\mathrm{~L} / 4$ \\
2 & $\mathrm{~L} / 4$ & $\mathrm{~L} / 8$ & $\mathrm{~L} / 16$ \\
3 & $\mathrm{~L} / 16$ & $\mathrm{~L} / 32$ & $\mathrm{~L} / 64$ \\
\hline
\end{tabular}

\section{Experimental Design}

Three experiments were conducted, which were identical in procedure except for luminance values used; these are shown in Table 1 by experiment number. Note that the lowest of the three intensity values for Experiment $\mathrm{j}$ becomes the highest of the three intensities of Experiment $j+1(j=1,2)$. This affords a test of replicability for these luminances and allows investigation of the effect of the context within which a stimulus appears (as the brightest or dimmest of the set).

Each experiment consisted of 6 experimental sessions. Essentially, each experimental session consisted of a sequence of simple RT trials about $3.5 \mathrm{sec}$ apart, with rest periods between each block (32). Within each block, 25\% (8) were catch trials (no stimulus). Over the remaining $75 \%$ (24) trials, stimulus duration and luminance varied randomly: permutations of the 32 stimuli ( 3 . 8 luminance-duration combinations plus 8 catch trials) were selected randomly and presented to the S. Altogether, within each session, there were 360 stimulus trials and 120 catch trials, or 15 observed RTs for each of the 24 data points (means) per session. Each experiment of 6 sessions therefore consisted of 2,160 observed RTs from stimulus trials and 720 catch trials, with each mean RT data point an average of 90 RTs. To determine stimulus intensity, the three stimulus fields were first equated for intensity to $15 \mathrm{fL}$ and the neutral density filters inserted into fields of the tachistoscope. Within each session, one-third (6) of the blocks of 32 trials each were run with a given filter-field assignment. To control for a possible effect of differences in stimulus characteristics of the three fields, the filter-field assignment (i.e., order) was shifted according to a Latin square design described below. Each session then consisted of 3 such orders of 6-block sets of trials, with the first block of each order discarded as warm-up. This left each session consisting of 3 orders by 5 blocks by 24 stimulus trials, for a total of 360 stimulus trials per session.

\section{Analysis of Variance Design}

In order to control for possible differences in stimulus characteristics due to field differences, the filter sets corresponding to, for example, $\mathrm{L} / 4, \mathrm{~L} / 2$, and $\mathrm{L}$, as referred to below, were rotated between fields each third part of a session. This procedure also made possible a test for order effects as well as field effects. Data points of each three successive Latin squares (three sessions) were arranged to comprise 3 orders by 3 fields by 3 luminances. The resultant so-called "complete block" was then replicated twice, yielding a total of six sessions per experiment, allowing an analysis of variance. The analysis, technically termed a "split plot" (Kempthorne, 1952), was designed to test for the effects of field $(F)$, order $(O)$, luminance (L), and duration (D). Recall that of the six sets of 32 trials collected in each one-third session, the first was discarded as warm-up, the remaining five kept for analysis. Each set of trials consisted of 24 RTs, one to each of the $3 \times 8$ luminance-duration stimulus combinations. Catch trial latencies were not included in the analysis. Finally. the analysis of 

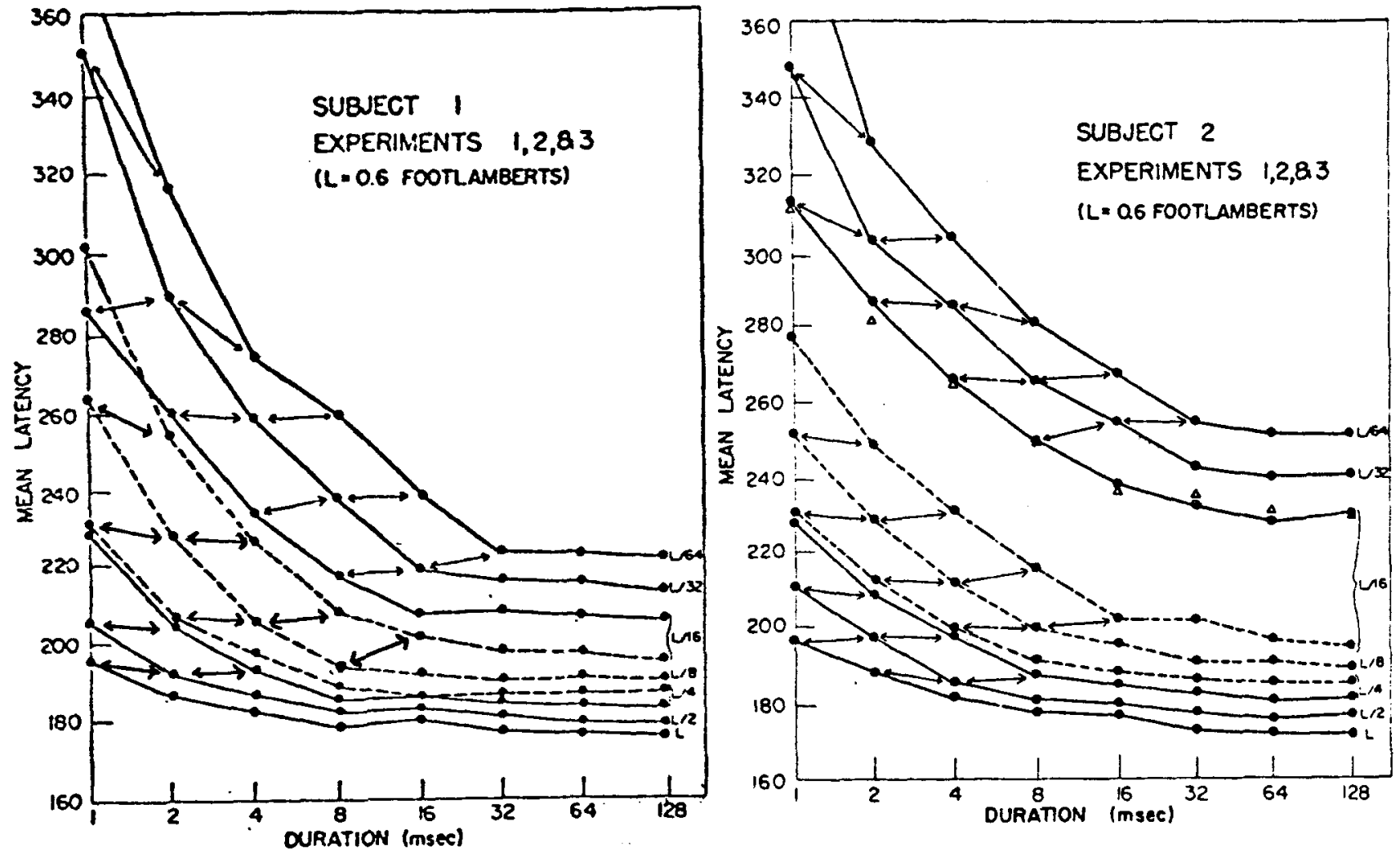

Figs. 2 and 3. The lines, solid, dotted, and solid, represent mean RTs of both Ss for Experiments 1, 2, and 3, respectively. Luminance parameters on the right, which decrease successively by a factor of $1 / 2$, may be converted to footlamberts via Table 1 . Open triangles for $S 2$ are $\mathrm{L} / 16$ points displaced upward by $33 \mathrm{msec}$ to show constant delay due to experiment context.

variance model assumption of no session-to-session trends was assured by providing enough practice days (about 6 per experiment) until mean RT curves had clearly reached asymptotic levels.

\section{EXPERIMENTAL REŚULTS}

Mean RTs as a function of stimulus duration (D) and intensity $(\mathrm{L})$ are shown in Figs. 2 and 3. Each data point represents an average of 90 RTs. Arrows are drawn between mean RTs for stimuli of equal energy ( $\mathrm{X} \times \mathrm{D}$ product); $B L$ holds to the degree that they are horizontal.

Note that for both Ss the same luminances, $\mathrm{L} / 4$ and $\mathrm{L} / 16$ gave rise to different latencies, depending on whether they were the highest or lowest intensities of the experiment. This seems to be a constant addition with respect to duration (D). This is shown most clearly in the case of $\mathrm{L} / 16$ for $\mathrm{S} 2$ (see caption). Here it appears that a constant of $33 \mathrm{msec}$ has been added to mean RTs as a result of the context of Experiment (E) 3. We suggest below that this may be due to a greater degree of dark adaptation with lower luminances.

It should be noted that this constant upward displacement appears in the same experiment (S 2, E 3) in which the probability of FAs has been reduced through practice over that of other experiments. For this experiment in particular, the required accuracy $-4 \%$
FAs-was at a cost of considerably increased latencies. Standard deviations (SDs) of RTs are shown in Figs. 4 and $S$ for Ss 1 and 2, respectively.

Bloch's law (BL) appears to hold approximately for mean RTs to the range of luminances examined here. This is indicated in Figs. 2 and 3 by the approximately horizontal arrowed lines between mean RTs to stimuli of equal $L \times D$ produce, except for the shortest durations and lowest intensities. It should be noted in connection with this, however, that a small constant error or variance in stimulus duration or luminance would be magnified in this region because of the logarithmic scales used for these variables. To show in another way the goodness of fit of BL, mean RTs have been replotted as a function of $L \times D$ product for Experiments 1-3, respectively, in Figs. 6-8.

Table 2

Estimated False Alarm and Miss Rates

\begin{tabular}{llll}
\hline $\mathrm{S}$ & $\mathrm{E}$ & $\mathrm{p}$ (FA) & $\mathrm{p}$ (Miss) \\
\hline \multirow{3}{*}{1} & 1 & .0167 & 00 \\
& 2 & .0431 & 0 \\
& 3 & .0319 & $.2 @(\ell, \mathrm{d})=(\mathrm{L} / 64,1)$ \\
& 1 & .025 & 0 \\
& 2 & .0347 & 0 \\
& 3 & .0194 & $.2 @(\ell, \mathrm{d})=(\mathrm{L} / 64,1)$ \\
\hline
\end{tabular}



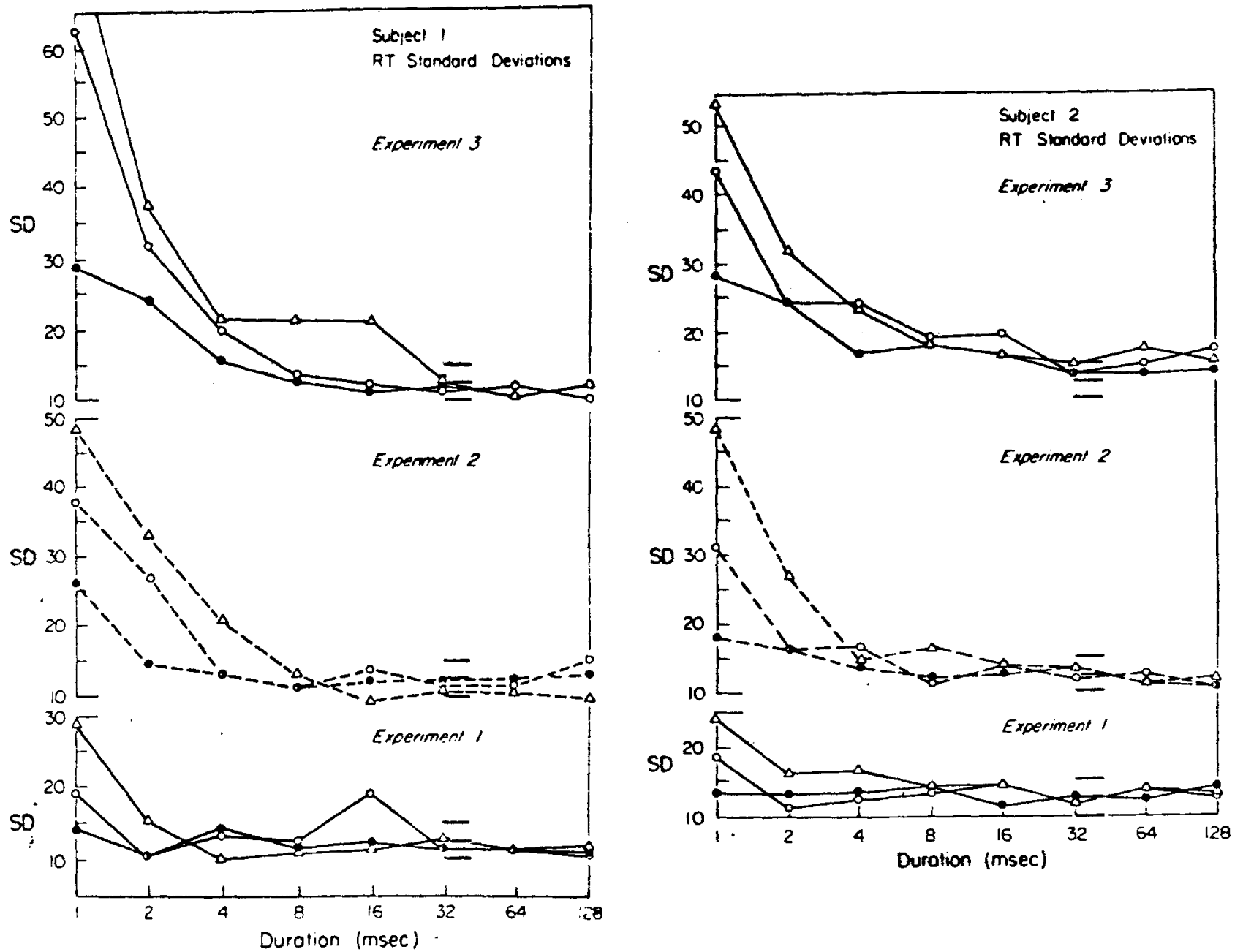

Figs. 4 and 5. Within each experiment, RT SDs to highest, medium, and lowest luminances (e.g., L, L/2, and L/4) are represented by filled circles, open circles, and open triangles, respectively. To facilitate comparison of asymptotic SDs across experiments, three short horizontal lines have been drawn near $d=32 \mathrm{msec}$ at $10,12.5$, and $15 \mathrm{msec}$. There appears to be relatively little change in observed asymptotic SDs even across experiments and between Ss.

The interesting question of whether BL holds for RT standard deviations is more difficult to answer; it appears, from examination of Figs. 4 and 5 that such a conclusion is not supported, i.e.. RT SDs to stimuli of the same $L \times D$ product are not in general similar enough to justify this conclusion. They are similar enough, however, to suggest that a more carefully conducted experiment with more precise stimulus generating equipment might indeed show that BL holds for means and variances, and perhaps even for RT distributions.

The analysis of variance for the six experiments showed that, in general. the only significant source of variance was due to luminance, duration, and their interaction. All were significant at better than the .001 level. Some higher order interaction appeared in Experiment 1 data, however. as well as an effect of field and order significant at the .05 level. The importance of this analysis is that it shows that effects due to field and order were negligible in general, and that the most significant feature of the data is the strong interaction between stimulus intensity and duration. The latter is, of course, evident in Figs. 2 and 3 and 6.8, where the good fit of $\mathrm{BL}$ itself indicates strong interaction between these variables.

\section{THEORY: TEMPORAL INTEGRATION MODELS FOR VISUAL REACTION TIME}

Our goal is to develop a model that can account for the relationships between RT and stimulus duration shown in Figs. 2-5. We begin with the well-known assumption that simple RT (the total delay between stimulus onset and S's response) is the sum of two components: $T_{d}$, the time between stimulus onset and detection; and $T_{r}$. the time between detection and response:

$$
R T=T_{d}+T_{r} .
$$


Meon RT os a Function of LXD Product
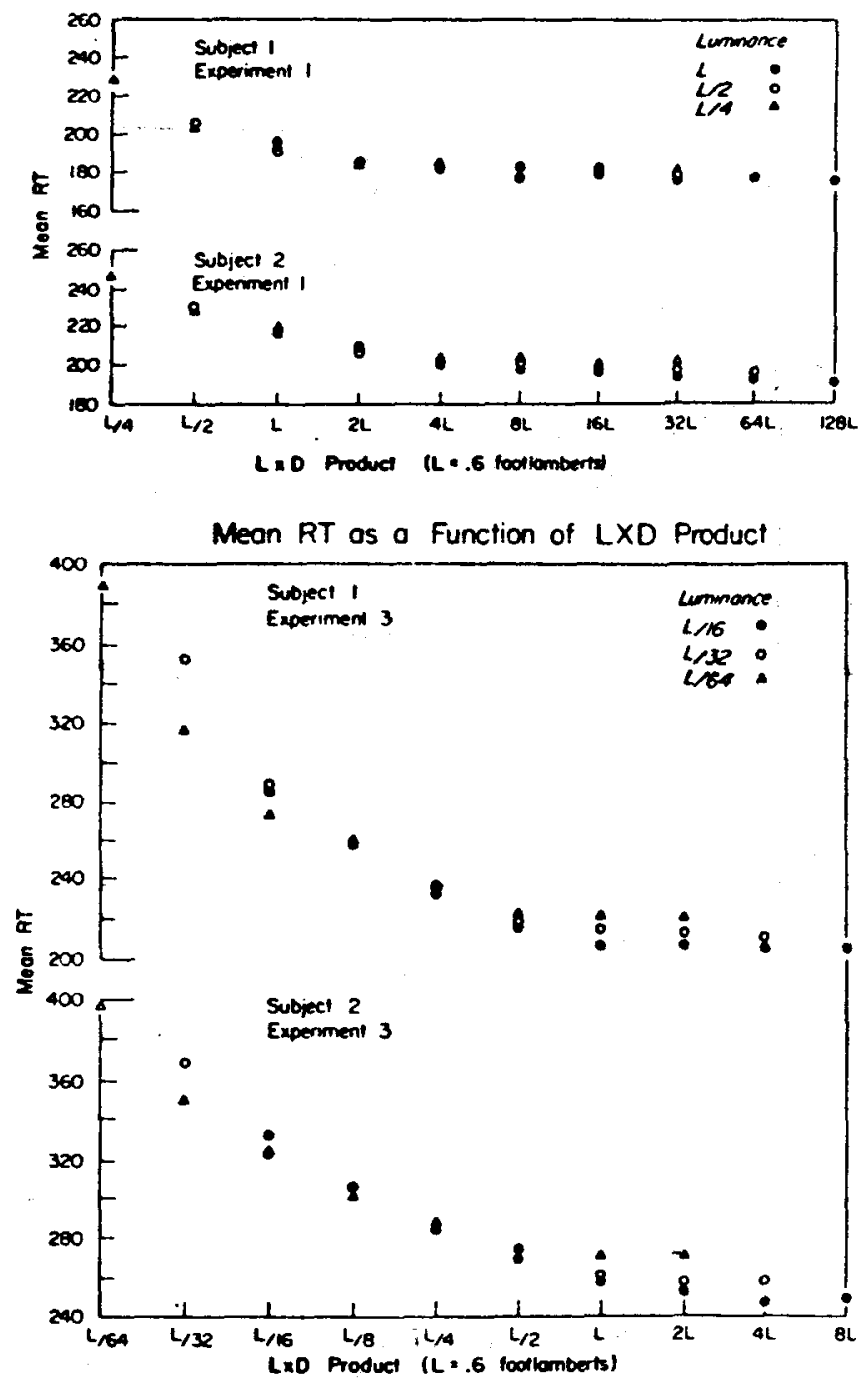

The temporal integration (TI) model, we now consider assumes that detection time, $T_{d}$, is the time required for the time integral of a nonnegative function $v(t)$ to reach some fixed criterion, $c$. The function, $v(t)$, is called the "visual response function" (VRF); its specific form depends on the visual stimulus. We assume $v(t)=0$ before stimulus onset, i.e., for $t=0$, let

$$
V(t)=\int_{0}^{t} v(t) d t .
$$

Then detection occurs when $V(t)=c$, and $T_{d}$ is the instant when this occurs, i.e.,

$$
\mathrm{V}\left(\mathrm{T}_{\mathrm{d}}\right)=\mathrm{c} .
$$

Thus

$$
R T=V^{-1}(c)+T_{r} .
$$

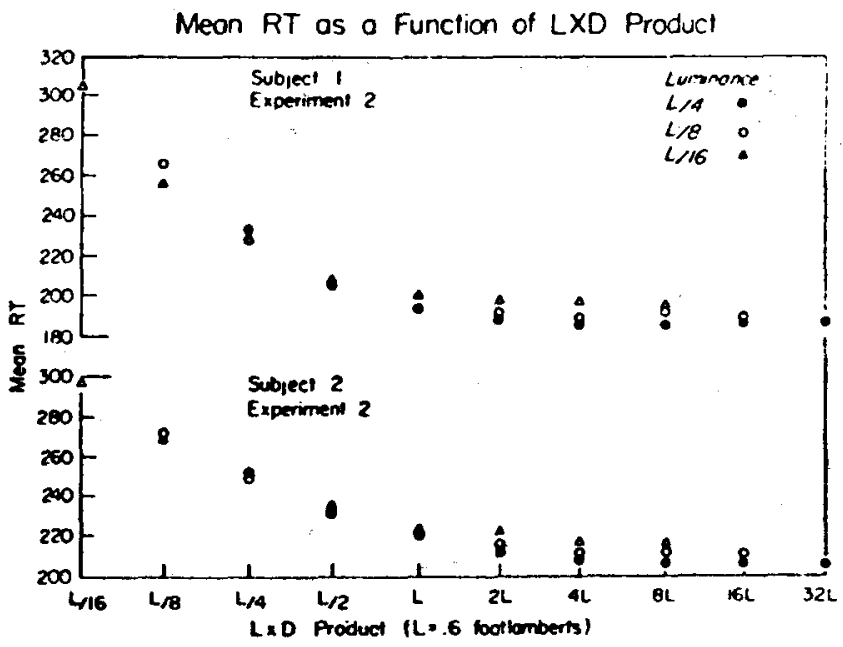

Figs. 6-8. Mean RTs and a function of $L \times D$ product support the earlier assertion that Bloch's law holds approximately.

For the purposes of this paper, it is assumed that for a given $S$ and a given set of experimental conditions, all of the model parameters are fixed, i.e., $c$ and $T_{r}$ are constants, and $v(t)$ is the same function on every trial involving the same stimulus. Note, however, that the model parameters may vary from experiment to experiment, and from $S$ to $S$. We acknowledge that this is an idealized model, and it may be desirable later on to consider some of these parameters as random variables which assume different values from trial to trial.

\section{THE EXPONENTIAL DECAY MODEL}

To obtain quantitative predictions from the Tl model, we need to assume an explicit form for the VRF. In the present application of the model, the visual stimulus is always assumed to be a square wave with intensity (i.e., luminance), $\ell$, presented at time zero and having duration $\mathrm{d}$. To indicate explicitly the dependence of the 
VRF on $d$ and $\ell$. we write $v(t ; d, \ell)$. The exponential decay model we now consider assumes that

$$
v(t ; d, \ell)= \begin{cases}0 & \text { for } t \leqslant \epsilon_{\ell}\left(\epsilon_{\ell} \geqslant 0\right) \\ \lambda_{\ell} & \text { for } \epsilon_{\ell}<t \leqslant d \\ \lambda_{\ell} e^{--r} l_{\ell}^{(t-d)} & \text { for } d \leqslant t\end{cases}
$$

In other words, the VRF corresponding to a square-wave flash with intensity $\ell$ and duration $d$ begins as a square wave with amplitude $\lambda_{\ell}$ at $t=\epsilon$, is maintained until time $d$, and then decays exponentially following offset of the flash (see Fig. 9). Of course, there may be a lag similar to $\epsilon$ before decay begins, but as long as $\epsilon$ is small we will omit consideration of it for simplicity. The parameter $r_{8}$ determines the rate of decay: at time $d+r_{Q}{ }^{-1} \log _{e}(2)$, the VRF will have decayed to $\lambda_{\ell} / 2$. The three parameters, $\epsilon_{\ell}, \lambda_{\ell}$, and $\mathrm{r}_{\ell}$ are all assumed to depend on the flash intensity, $\ell$. When the criterion parameter. c, and the detection-to-motor-response parameter, $T_{r}$, are included, there are five free parameters altogether in this exponential decay version of the TI model. However, we show below that for any fixed value of $\ell$, the number of parameters that must be estimated in order to generate the predicted relationship between flash duration and RT is actually three. Put another way, the exponential decay model really involves only three "identifiable" parameters (Greeno \& Steiner, 1964).

Detection occurs when the integral of $\mathrm{v}(\mathrm{t})$ reaches the criterion, c. For the model given by Eq. 4, we have

$$
V(t ; d, l)= \begin{cases}0 & t \leqslant \epsilon_{\ell} \\ \lambda_{\ell}\left(t-\epsilon_{\ell}\right) & \epsilon_{\ell}<t \leqslant d \\ \lambda_{\ell}\left(d-\epsilon_{\ell}\right)+\frac{\lambda_{\ell}}{r_{\ell}}\left[1-e^{-r} r^{(t-d)}\right] & d<t .\end{cases}
$$

The quantity $\mathrm{V}(\infty ; \mathrm{d}, l)$ can be thought of as the "total (integrated) response" generated by the flash. If $\mathrm{V}(\infty ; \mathrm{d}, \ell)<\mathrm{c}$, the flash will not be detected. It is natural to introduce a parameter $\delta_{\ell}$ corresponding to the "threshold duration" for a flash of intensity $\ell: \delta_{\ell}$ is the shortest duration for which such a flash is above threshold; i.e., $\mathrm{V}\left(\infty ; \delta_{\ell}, \ell\right)=$ c. Thus, from Eq. 5 ,

$$
\delta_{\ell}=\frac{c}{\lambda_{\ell}}+\epsilon_{\ell}-r_{\ell}^{-1} .
$$

At the other extreme, we note that if $d$ is sufficiently long, $\mathrm{V}(\mathrm{d} ; \mathrm{d}, \mathrm{l})>\mathrm{c}$ and detection occurs during the flash itself. For all such durations, the detection latency will be the same, i.e., if $V(d ; d, \ell)>c$, then

$$
T_{d}=\frac{c}{\lambda_{Q}}+\varepsilon_{Q}
$$
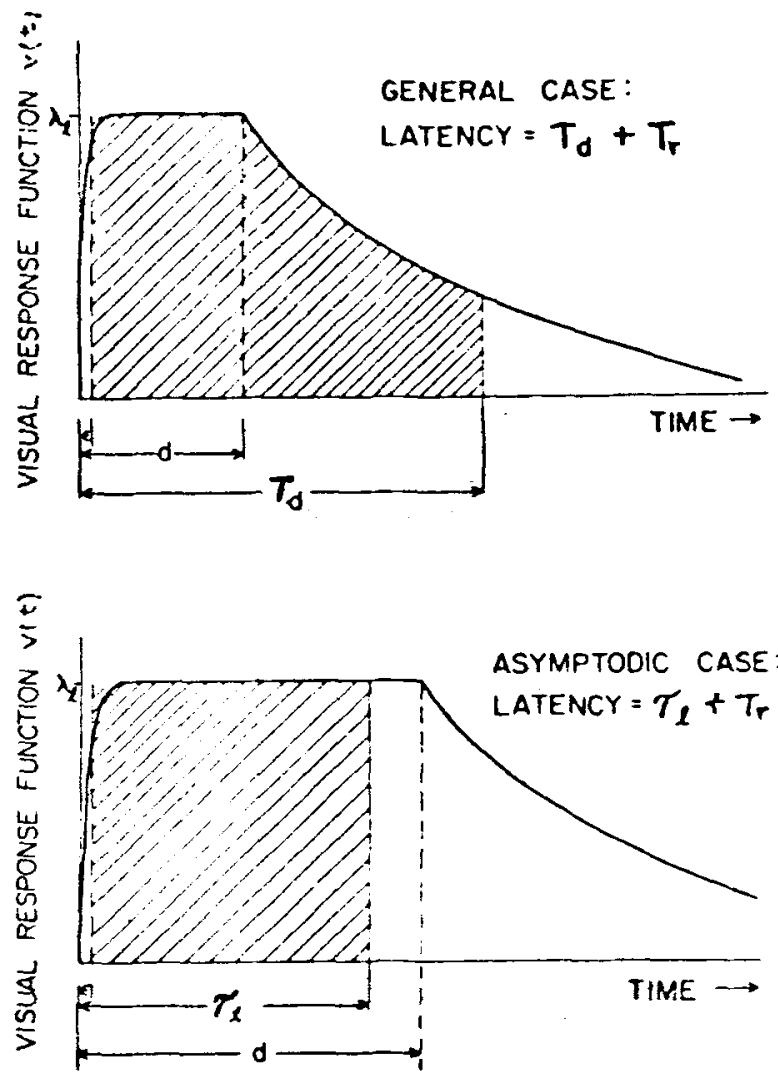

Fig. 9. The visual response function (VRF) depends on light intensity, $\ell$ (via $\lambda_{\ell}$ and $r_{\ell}$ ), and duration, $d$. The visual system simply integrates as much of it as is needed for detection. When $d>t$, the latency is no longer a function of $d$, but of $\lambda_{l}$ alone.

It is convenient to introduce a parameter $\tau_{\ell}$ corresponding to the shortest duration for which $\mathrm{V}(\mathrm{d} ; \mathrm{d}, \ell)>\mathrm{c}$. In this case, $\mathrm{V}\left(\tau_{\ell} ; \tau_{\ell}, \ell\right)=\mathrm{c}$, i.e., $\lambda_{\ell}\left(\tau_{\ell}-\epsilon_{\ell}\right)$ $=c$; hence

$$
\tau_{\ell}=\frac{\mathrm{c}}{\lambda_{\ell}}+\epsilon_{\ell}
$$

$\tau_{\ell}$ can be thought of as the "critical duration" for flashes with luminance $\ell$; all flashes with $\mathrm{d}>\tau_{\ell}$ will have the same latency (i.e., increasing the duration beyond $\tau_{\ell}$ does not lead to any further decrease in RT).

Now, if $\delta_{\ell}<\mathrm{d}<\tau_{\ell}$, the flash will be detected at some point during the exponential decay portion of its VRF. To determine $T_{d}$ in this case we solve the third line of Eq. 5 for $V\left(T_{d}: d . l\right)=c$ and obtain

$$
\mathrm{T}_{\mathrm{d}}=\mathrm{d}-\frac{1}{\mathrm{r}_{\ell}} \log _{\mathrm{e}}\left[1-\mathrm{r}_{\ell}\left(\frac{\mathrm{c}}{\lambda_{\ell}}+\epsilon_{\ell}-\mathrm{d}\right)\right] \text {. }
$$

Finally, then, combining Eqs. 7 and 9, and using Eq. 8 to substitute for $c / \lambda_{\ell}+\epsilon_{Q}$, we have the following general expression of the detection latency $T_{d}$ of a flash with Luminance $\ell$ and duration $\mathrm{d}$ : 
Table 3

Parameter Estimates from Method 1 ( $\mathrm{L}=.6 \mathrm{fL}$, All Other Values are in Milliseconds)

\begin{tabular}{|c|c|c|c|c|c|c|}
\hline$S$ & $\begin{array}{c}\text { Experi- } \\
\text { ment }\end{array}$ & $f$ & $\mathrm{~T}_{\mathrm{I}}$ & $\dot{\delta}_{\ell}$ & $\hat{\mathrm{I}} \overline{\bar{Q}}$ & $\hat{\tau}_{\ell}=\dot{I}_{\ell}{ }^{1}+\dot{\delta}_{\ell}$ \\
\hline 1 & 1 & $\begin{array}{l}\mathrm{L} \\
\mathrm{L} / 2 \\
\mathrm{~L} / 4\end{array}$ & $\begin{array}{l}165.8 \\
167.3 \\
167.6\end{array}$ & $\begin{array}{l}.30 \\
.51 \\
.81\end{array}$ & $\begin{array}{l}10.42 \\
11.55 \\
14.04\end{array}$ & $\begin{array}{l}10.72 \\
12.06 \\
14.85\end{array}$ \\
\hline 1 & 2 & $\begin{array}{l}L / 4 \\
L / 8 \\
L / 16\end{array}$ & $\begin{array}{l}171.6 \\
169.6 \\
169.4\end{array}$ & $\begin{array}{l}.86 \\
.87 \\
.85\end{array}$ & $\begin{array}{l}13.25 \\
19.16 \\
26.16\end{array}$ & $\begin{array}{l}14.11 \\
20.03 \\
27.01\end{array}$ \\
\hline 1 & 3 & $\begin{array}{l}L / 16 \\
L / 32 \\
L / 64\end{array}$ & $\begin{array}{l}180.3 \\
180.3 \\
182.7\end{array}$ & $\begin{array}{l}.62 \\
.85 \\
.84\end{array}$ & $\begin{array}{l}25.16 \\
32.00 \\
37.62\end{array}$ & $\begin{array}{l}25.78 \\
32.85 \\
38.46\end{array}$ \\
\hline 2 & 1 & $\begin{array}{l}L \\
L / 2 \\
L / 4\end{array}$ & $\begin{array}{l}179.7 \\
183.6 \\
183.3\end{array}$ & $\begin{array}{l}0 \\
.49 \\
.50\end{array}$ & $\begin{array}{l}13.79 \\
13.82 \\
17.88\end{array}$ & $\begin{array}{l}13.79 \\
14.31 \\
18.38\end{array}$ \\
\hline 2 & 2 & $\begin{array}{l}L / 4 \\
L / 8 \\
L / 16\end{array}$ & $\begin{array}{l}187.0 \\
187.8 \\
189.0\end{array}$ & $\begin{array}{l}.46 \\
.47 \\
.38\end{array}$ & $\begin{array}{l}17.77 \\
22.06 \\
27.88\end{array}$ & $\begin{array}{l}18.23 \\
22.53 \\
28.26\end{array}$ \\
\hline 2 & 3 & $\begin{array}{l}L / 16 \\
L / 32 \\
L / 64\end{array}$ & $\begin{array}{l}219.8 \\
226.4 \\
233.6\end{array}$ & $\begin{array}{l}.28 \\
.56 \\
.56\end{array}$ & $\begin{array}{l}29.81 \\
32.38 \\
36.32\end{array}$ & $\begin{array}{l}30.09 \\
32.94 \\
36.88\end{array}$ \\
\hline
\end{tabular}

$$
T_{d}= \begin{cases}\infty(\text { no detection }) & \text { if } d \leqslant \delta_{\ell} \\ d-\frac{1}{r_{l}} \log _{e}\left[1-r_{\ell}\left(\tau_{l}-d\right)\right] & \text { if } \delta_{\ell}<d<\tau_{\ell}(10) \\ \tau_{l} & \text { if } \tau_{\ell} \leqslant d\end{cases}
$$

Since $R T=T_{d}+T_{r}$, the three parameters required to fit mean RT as a function of flash duration for a fixed value of $\ell$ are $T_{r}, \tau_{\ell}$, and $r_{\ell}$. Alternatively, we could express $T_{d}$ in terms of $r_{\ell}$ and the threshold duration $\delta_{\ell}$, using Eqs. 6 and 8:

$$
\mathrm{T}_{\mathrm{d}}= \begin{cases}\infty \text { (no detection) } & \mathrm{d} \leqslant \delta_{\ell} \\ \mathrm{d}-\frac{1}{\mathrm{r}_{\ell}} \log _{\mathrm{e}}\left[\mathrm{r}_{\ell}\left(\mathrm{d}-\delta_{\ell}\right)\right] & \delta_{\ell}<\mathrm{d}<\tau_{\ell}=\delta_{\ell}+\frac{1}{\mathrm{r}_{\ell}} \\ \delta_{\ell}+\frac{1}{\mathrm{r}_{\ell}} & \mathrm{r}_{\ell} \leqslant \mathrm{d}\end{cases}
$$

In either case, three parameters must be estimated from the data. It is worth noting that Eq. 10 provides a check on the assumption that $T_{r}$ is independent of the stimulus parameters: By subtracting the observed critical duration (i.e., the observed duration value at which the RT vs duration function becomes asymptotic) from the asymptotic latency, we obtain an estimate of $\left(\tau_{\ell}+T_{r}\right)-\tau_{\ell}=T_{r}$. These estimates should be roughly equal for all luminances used in any given experiment.

\section{PARAMETER ESTIMATION AND GOODNESS OF FIT}

Let $\mathrm{RT}(\mathrm{d}, \ell)$ denote mean $\mathrm{RT}$ as a function of duration for a stimulus with luminance $\ell$. Using Eq. 11, the ED model prediction for this function is

$$
\operatorname{RT}(\mathrm{d} ; \ell)= \begin{cases}\mathrm{T}_{\mathrm{r}}+\mathrm{d}-\frac{1}{\mathrm{r}_{\ell}} \log \left[\mathrm{r}_{\ell}\left(\mathrm{d}-\delta_{\ell}\right)\right] & \delta_{\ell}<\mathrm{d}<\tau_{\ell} \\ \delta_{\ell}+\mathrm{r}_{\ell}^{-1}+\mathrm{T}_{\mathrm{r}} & \tau_{\ell} \leqslant \mathrm{d} \quad(1 \mathrm{bb})\end{cases}
$$

We denote by $\mathrm{RT}(\infty ; \ell)$ the asymptotic latency (i.e., for $\mathrm{d}$ large). Then

$$
\begin{aligned}
\operatorname{RT}(\mathrm{d} ; \ell) & -\mathrm{RT}(\infty ; \ell) \\
= & \left(\mathrm{d}-\delta_{\ell}\right)-\mathrm{r}_{\ell}^{-1}-\mathrm{r}_{\ell}^{-1} \log \left[\mathrm{r}_{\ell}\left(\mathrm{d}-\delta_{\ell}\right)\right] .
\end{aligned}
$$

Empirically, Eq. 12 corresponds to the latency vs duration functions of Figs. 2 and 3 displaced down so as to be asymptotically zero. Estimates of the parameters $\delta_{\ell}$ and $\mathrm{r}_{\ell}^{-1}$ were obtained for each luminance in each experiment by means of a least-squares computer program which calculated the best fitting form of Eq. 12 for each case separately. The resulting estimates are shown in Table 3. The goodness of fit was excellent, but not surprising in view of the number of free parameters-seven. The table also shows estimates of $T_{r}$ for each case; these were obtained by subtracting $\hat{\delta}_{\ell}+\hat{T}_{\ell}-1$ from the observed asymptotic mean latency [i.e., the empirical counterpart of $\mathrm{RT}(\infty ; \ell)$ ]. Table 3 also includes an estimate of the critical duration $\tau_{\ell}$ for each case; these estimates are simply the sums $\hat{\delta}_{\ell}+\hat{r}_{\ell}-1$.

It will be noted that the estimates $\hat{\delta}_{\ell}$ in Table 3 are approximately constant within each experiment (except for S $1 E 1$ and S 2 E 1). This is also true of the estimates $\hat{T}_{\mathrm{r}}$ (except for S 2 E 3). However, it is quite clear that $\hat{T}_{r}$ changed considerably from experiment to experiment-as much as $40 \mathrm{msec}$ for $S 2$.

Because $\hat{T}_{r}$ and $\hat{\delta}_{\ell}$ seemed to be virtually constant within each experiment, a second method of parameter estimation was also applied to the data. In this method, all three curves from each experiment were fit simultaneously by a least squares program in which $\hat{\delta}$ and $\hat{T}_{r}$ were required to be the same for all three, while $\hat{I}_{\ell}$ was permitted to assume different values for different luminances. The resulting estimates are given in Table 4; goodness of fit is shown in Figs. 10 and 11. It can be seen that even with these restrictions, the fit is quite good-except perhaps for S2 E 3, where the discrepancies between $T_{r}$ estimates in Table 3 now have the effect of producing notable discrepancies between observed and predicted values at asymptote.

\section{THEORETICAL INTERPRETATION OF PARAMETER ESTIMATES}

The fact that $1 / r_{\ell}$ decreases with $\ell$ in Table 3 means that the half-life of the effect of a stimulus increases as its luminance decreases. This is rather surprising; one might have expected that a more intense stimulus would 

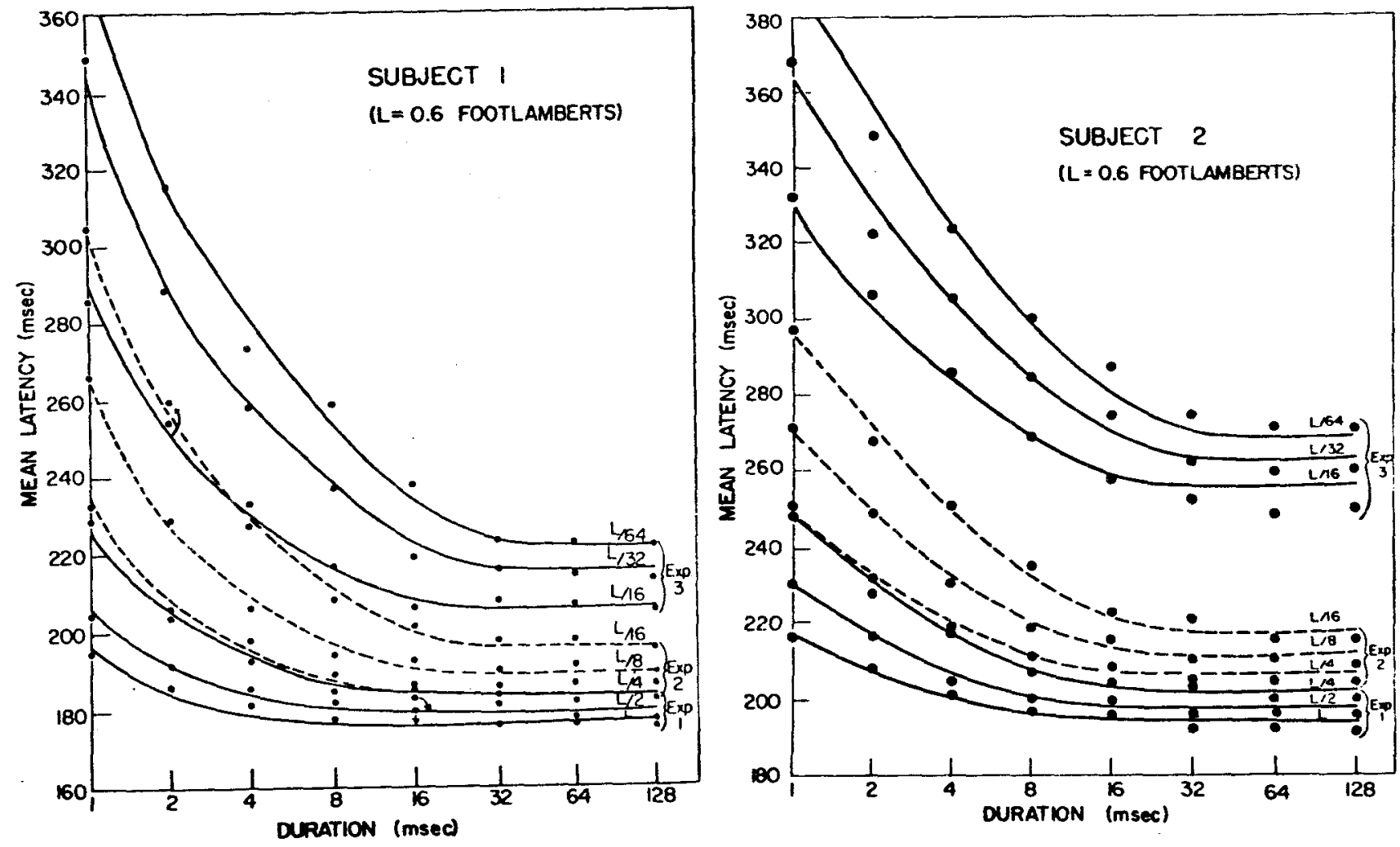

Figs. 10 and 11. Goodness of fit of the TI-ED model to the observed mean RTs.

have the effect of greater persistence, i.e., $v(t)$ requires a longer time to decay for any given fixed level.

A second surprising aspect of Table 3 is the fact that $\hat{\delta}_{\ell}$ seems to be roughly constant over $\ell$ in most of the experiments. Since $\delta_{\ell}$ is the threshold duration, we would expect it to increase as luminance decreases-the less intense the stimulus, the longer it must last to reach threshold. Perhaps it is sufficient that the $\hat{\delta}_{\ell}$ values are

Table 4

Parameter Estimates from Method 2*

\begin{tabular}{|c|c|c|c|c|c|c|c|c|}
\hline $\mathrm{S}$ & $\begin{array}{c}\text { Experi- } \\
\text { ment }\end{array}$ & e & $\dot{\mathrm{T}}_{\mathrm{I}}$ & $\hat{\delta}_{\ell}$ & $\hat{r}_{\bar{\ell}}^{-1}$ & $\hat{\tau}_{\ell}=\hat{\mathrm{r}}_{\bar{Q}}{ }^{-1}+\hat{\delta}_{\ell}$ & $1 / \hat{\alpha}$ & $\hat{\beta}$ \\
\hline 1 & 1 & $\begin{array}{l}L \\
L / 2 \\
L / 4\end{array}$ & 168.2 & .74 & $\begin{array}{r}7.93 \\
10.20 \\
14.30\end{array}$ & $\begin{array}{r}8.67 \\
10.94 \\
15.04\end{array}$ & 6.4 & .43 \\
\hline 1 & 2 & $\begin{array}{l}L / 4 \\
L / 8 \\
L / 16\end{array}$ & 167.8 & .83 & $\begin{array}{l}14.65 \\
20.20 \\
26.84\end{array}$ & $\begin{array}{l}15.48 \\
21.03 \\
27.67\end{array}$ & 6.6 & .44 \\
\hline 1 & 3 & $\begin{array}{l}L / 16 \\
L / 32 \\
L / 64\end{array}$ & 182.2 & .82 & $\begin{array}{l}22.66 \\
31.97 \\
38.12\end{array}$ & $\begin{array}{l}23.48 \\
32.79 \\
38.94\end{array}$ & 7.0 & .38 \\
\hline 2 & 1 & $\begin{array}{l}L \\
L / 2 \\
L / 4\end{array}$ & 183.2 & .44 & $\begin{array}{l}11.11 \\
14.12 \\
18.28\end{array}$ & $\begin{array}{l}11.55 \\
14.56 \\
18.72\end{array}$ & 9.5 & .36 \\
\hline 2 & 2 & $\begin{array}{l}L / 4 \\
L / 8 \\
L / 16\end{array}$ & 189.0 & .44 & $\begin{array}{l}17.23 \\
21.89 \\
27.58\end{array}$ & $\begin{array}{l}17.67 \\
22.33 \\
28.02\end{array}$ & 9.3 & .34 \\
\hline 2 & 3 & $\begin{array}{l}L / 16 \\
L / 32 \\
L / 64\end{array}$ & 229.5 & .53 & $\begin{array}{l}25.35 \\
31.88 \\
37.91\end{array}$ & $\begin{array}{l}25.88 \\
32.41 \\
38.44\end{array}$ & 10.1 & .29 \\
\hline
\end{tabular}

${ }^{*} L=.6 \mathrm{fL}, \beta$ is a dimensionless constant, and all other values are in milliseconds. $\alpha$ and $\beta$ are optimal constants for power function $r_{Q}=\alpha Q^{\beta}$. 


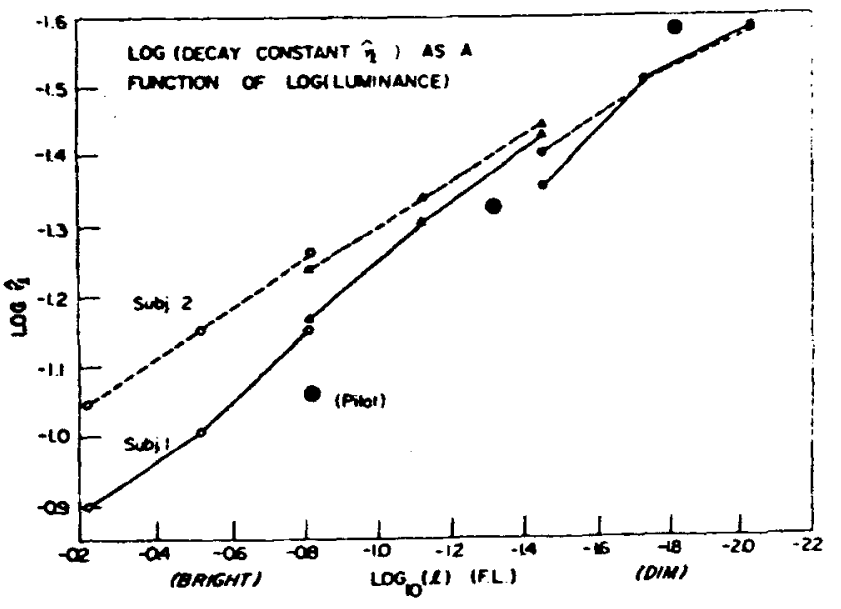

Fig. 12. Logarithms of parameter estimates $\dot{i}_{\ell}$ as a function of $\log (\ell)$. The points fall in a nearly linear pattern with estimated slopes near $1 / 3$, except for the pilot experiments (large dots) with a slope of nearly $1 / 2$.

small, i.e., less than $1 \mathrm{msec}$; it may be unrealistic to expect finer resolution from RT data of the sort considered here.

A natural question in the context of the exponential decay model is how the parameters $I_{\ell}$ and $\lambda_{\ell}$ are related to flash luminance. Figure 12 shows a plot of $\log \left(\hat{\mathrm{r}}_{\ell}\right)$ vs $\log (\ell)$. The relationship is approximately linear for both $S s$ in all experiments; best-fitting slope parameters are given in Table 4. A linear relationship between $\log \left(\mathrm{r}_{\ell}\right)$ and $\log (\ell)$ means that $r_{\ell}$ is a power function of $\ell$, i.e.,

$$
\hat{\mathrm{r}}_{\ell}=\alpha Q^{\beta} \text {. }
$$

Table 4 shows that $\hat{\beta}$ here was consistently in the neighborhood of .35 , roughly the same value found for brightness as a function of luminance (cf. Stevens, 1966). Since $\tau_{\ell}=c / \lambda_{\ell}+\epsilon_{\ell}=\delta_{\ell}+1 / \mathrm{r}_{\ell}$, and $\hat{\delta}_{\ell}$ here was small, it follows that $c / \lambda_{l}+\epsilon_{\ell}$ and $1 / \hat{r}_{\ell}$ were approximately equal here, and consequently $\left(\mathrm{c} / \lambda_{\ell}+\right.$ $\left.\epsilon_{\ell}\right)^{-1}$ was also a power function of $\ell-$ to a good approximation-with the same exponent as $\hat{r}_{\ell}$. If $\epsilon_{\ell} \equiv 0$, this means that $\lambda_{\ell}$, the amplitude of VRF, is a power function of luminance, with exponent in the range .3 to .5. This seems a reasonable result, since we should expect brightness to be proportional to the maintained level of the VRF. Or, put more realistically, if we were to extend the model to account for brightness phenomena, this would be a natural first assumption, and so it is gratifying to see that it is not disconfirmed by our parameter estimates.

\section{SOME CONCLUDING REMARKS ON THE TI-ED MODEL}

We have indicated above an important limitation of the TI-ED model: As it stands, it is a deterministic model and does not provide any account of the stochastic character of RTs. Although an extensive development of a stochastic version of the model is beyond the scope of this paper, there are preliminary indications that assuming $c$ to be a random variable provides a good quantitative fit to the observed variances and explains the approximate invariance of the $\hat{\delta}$ within each experiment. At this time, however, we limit ourselves to the qualitative argument that if $c, \lambda_{\ell}$, or $\tau_{\ell}$ is a random variable, the variances will be inflated for small $d$; then the observed variances are at least qualitatively compatible with the model.

Although our finding that the half-life [the time required for $v(t)$ to decay to half its original value] increases with decreasing luminance seems paradoxical, a recent electrophysiological study by Levick and Zacks (1970) suggests that such "decay" may be a natural consequence of the way retinal ganglion cells respond to stimulus intensity changes for short light flashes. This study (see Fig. 2, p. 682) suggests that as stimulus intensity increases (for fixed duration flashes), the ganglion cell response actually becomes more sharply localized in time rather than becoming more prolonged.

Finally, the apparent changes in $T_{r}$ from experiment to experiment may be the result of changes in dark adaptation level. Since different stimul us intensities were used in each of the three experiments, the effective dark adaptation level was greatest in E 3, next greatest in E 2, and least in E 1. There is considerable evidence, both physiological (Levick \& Zacks, 1970) and psychophysical (Standing, Dodwell, \& Lang, 1968) showing that visual latency increases with the amount of dark adaptation, so that on this basis we would expect the longest "baseline" latency in E 3 and the shortest in E 1, which was found. If dark-adaptation level could somehow be held constant across all conditions, changes in $T_{r}$ from one condition to another could then be attributed to changes in the criterion, $c$. The lengthy procedure required, however, might be impractical in view of the large number of observations necessary for parameter estimation.

\section{PILOT STUDY}

A pilot study had been conducted using almost exactly the same procedure as in the experiments reported above. The only important difference was that the luminances used were $.15, .0475$, and $.015 \mathrm{fL}$, expanding the luminance range from 4 to 10 . The $S$ was the author. Observed and predicted mean RTs for the pilot experiment are shown in Fig. 13. To see that BL does not hold in this case, displace the observed data points of the figure right or left by $1 / 2 \log _{10}$ units, or $1.67 \log _{2}$ units. The reduction in intensity by a factor of 3.1 when exactly matched by a corresponding increase in stimulus duration of $3.1=21.67$ leads to RT predictions which consistently fall below observed mean RTs by $10.15 \mathrm{msec}$, or about the difference in estimated critical durations. 
Nevertheless, the ED model does an excellent job of accounting for the mean RTs. The parameter estimates $\hat{\mathbf{r}}_{\ell}$, however, depart from those obtained in the main experiments in that, for the pilot study, $\hat{\mathrm{r}}_{\ell}=\ell^{.5}$ vs $\hat{\mathrm{r}}_{\ell}=$ Q.33 for the major experiments (cf. Fig. 12-large dots). We see therefore that the conclusion that Bloch's law holds must be conditioned by the observation that it fails when the range of luminances used is as large as 10 (i.e., $\ell_{0}$ to $10 \ell_{0}$ ). Nevertheless, the ED model accounts well for the data (cf. Fig. 13).

One possible cause of the failure to obtain $\mathrm{BL}$ in the pilot experiment could be artifactual trial-to-trial light

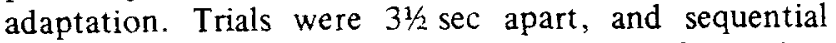
effects would be most severe in wide ranges of intensity.

\section{THE RELATIONSHIP BETWEEN BLOCH'S LAW, THE POWER LAW FOR SIMPLE RT, AND THE ED MODEL}

We saw above that within each main experiment involving a fixed set of luminances and durations, BL held for mean RTs to a close approximation (cf. Figs. 6-8). Now, a natural question at this point is whether the ED model, with parameters $r_{\ell}$ and $\lambda_{\ell}$ constrained as in $\hat{r}_{\ell}=Q^{\beta}$, actually implies BL will hold exactly. It is easily shown, even with $\delta=\epsilon$, so that $\mathrm{r}_{\varnothing}=$ $\lambda_{\ell} / \mathrm{c}$, that it does not (proof omitted for brevity). However, it can be shown that when $\beta \doteq 1 / 3$, then the ED model with $r_{\ell}$ and $\lambda_{\ell}$ both proportional to $\ell^{\beta}$ does imply a close approximation to BL over a limited range of $\ell$ values -in particular. $\ell$ values such that $\ell .33-1$ and $\log _{10}(\ell)$ are approximately equal. The ranges of $\ell$ values used in each of the main experiments were all of the form $\ell_{0}<\ell<4 \ell_{0}$. so the necessary approximations were valid there. Consequently, even though the ED model does not strictly imply $\mathrm{BL}$, it is compatible with the approximate validity of that law found in the present experiments.

\section{SUMMARY}

In the foregoing, we have attempted to answer the question of whether Bloch's law (BL) holds for RTs to suprathreshold light stimuli. Earlier research on this subject had been limited to three studies, of which two had concluded that BL did not, in general, hold for RTs. The present research, on the other hand, indicated the opposite. For the limited range of luminances-all suprathreshold-that we investigated, BL did hold to a very good approximation. The results of the experiments were orderly enough to be fit by a simple mathematical model. A special case of the model-called the temporal integration-exponential decay (TI-ED) model-provides a very good fit for the mean RTs of these experiments.

According to the model, RTs to a square-wave flash are determined by the time required to integrate a "visual response function" (VRF) generated by that flash. For the TI-ED model, the VRF rises instantly to

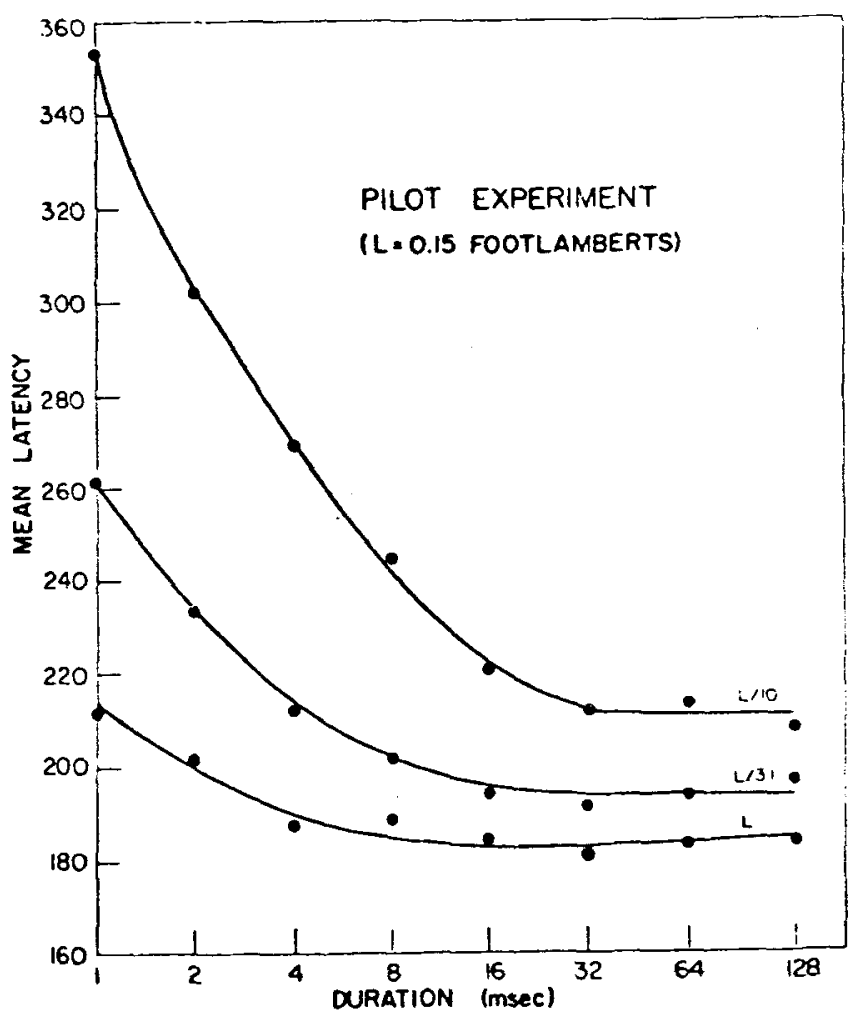

Fig. 13. Goodness of fit of a pilot experiment using luminances $L, L / 3.2$, and $L / 10$, where $L=0.15 \mathrm{fL}$.

amplitude $\lambda_{\ell}$ with the onset of the flash and remains at that level until flash offset. The VRF then decays exponentially with a time constant, $r_{\ell}^{-1}$. Both $r_{\ell}^{-1}$ and $\lambda_{\ell}$ depend on the stimulus intensity, $\ell$.

\section{REFERENCES}

Adrian, E. D., \& Matthews, R. The action of light on the eye. Part I. The discharge of impulses in the optic nerve and its relation to the electric changes in the retina. Journal of Physiology, 1927, 63, 378-414.

Armington, J. C., Tepas, K. I., Kropfl, W. J., \& Hengst. W. H. Summated retinal potentials. In E. P. Horne and M. A. Whitcomb (Eds.), Vision research papers. Washington, D.C: National Academy of Sciences, National Research Council. 1960. P. 112.

Bloch, A. M. Experiences sur la vision. Soc. Biol. Mem. (Paris). $1885.27 .493-495$

Greeno, J. G., \& Steiner. T. E. Markovian processes with identifiable states: General considerations and application to all-or-none learning. Psychometrika, 1964, 29, 309-333.

Grossberg, M. The latency of response in relation to Bloch's law at threshold. Perception \& Psychophysics, 1968, 4, 229-232.

Hanes. R. M. The construction of subjective brightness scales from fractionation data: A validation. Journal of Experimental Psychology, 1949, 39. 719-728.

Hartline. H. K. Intensity and duration in the excitation of a single photoreceptor unit. Journal of Cellular Comparative Physiology, 1934, 5. 229-247.

Hopkinson, R. G. Light energy and the brightness sensation. Nature, 1956, 178, 1065-1066.

Kempthorne, O. Design and analysis of experiments. New York Wiley, 1952. Pp. 370-384. 
Kietzman, M., \& Gillam, B. Visual temporal integration and simple reaction time. Perception \& Psychophysics, 1972, 11, 333-340.

Levick, W. R.. \& Zacks, J. L. Responses of cat retinal ganglion cells to brief flashes of light. Journal of Physiology, 1970, 206. 677-700.

Lewis. M. F. Magnitude estimation and reaction time as functions of flash luminance and duration in the fovea. Unpublished doctoral dissertation, Columbia University, 1964. (Cited in Grossberg, 1968.)

Liang, T., \& Pieron, H. De l'influence specifique des eclairments monochromatiques sur le retard de la sensation visuelle. Comptes Rendus Hebdomadaire Academie Scientifique, Paris, 1945, 56. 221.

Onley, J. W. Light-adaptation and the brightness of brief foveal stimuli. Joumal of the Optical Society of America, 1961, 51, $667-673$.

Pieron, $H$. Nouvelles recheres sus l'analyse du temps de latence sensorielle et sur la qui relie le temp a l'intensité d'excitation. Année de Psychologie, 1920, 22, 58-142.

Pieron. H. The sensations. New Haven, Conn: Yale University Press. 1952.

Raab, D. H. Magnitude estimation of the brightness of brief foveal stimuli. Science, New York, 1962, 135, 42-44.

Raab, D.. \& Fehrer, E. The effect of stimulus duration and luminance on visual reaction time. Journal of Experimental Psychology, 1962, 64, 326-327.
Sperling, H. G., \& Jolliffe, C. L. Intensity-time relationship at threshold for spectral stimuli in human vision. Journal of the Optical Society of America, 1965, 55, 191-199.

Standing, L. G.. Dodwell, P. C., \& Lang. D. Dark adaptation and the Pulfrich effect. Perception \& Psychophysics, 1968, 4. 118-120.

Stevens, S. S. Duration, luminance, and the brightness exponent. Perception \& Psychophysics, 1966, 1, 96-100.

Stevens, S. S.. \& Galanter, E. H. Ratio scales and category scales for a dozen perceptual continua. Journal of Experimental Psychology, 1957, 54, 377-411.

Sticht, T. Effects of intensity and duration on the latency of response to brief light and dark stimuli. Journal of Experimental Psychology, 1969, 80, 419-422.

Teichner, W., \& Krebs, M. Laws of simple visual reaction time. Psychological Review, 1972, 79, 344-358.

Vaughan, H. G., Jr. Perceptual and physiologic significance of visual evoked responses recorded from the scalp in man. Proceedings of the Third International Conference of the Society for Clinical Electroretinography, 1964, 203-223.

Vaughan, H. G., Jr., Costa, L. D., \& Gilden, L. The functional relation of visual evoked response and reaction time to stimulus intensity. Vision Research, 1966, 6, 645-656.

(Received for publication November 6, 1972; revision received May $13,1973$. 